\title{
新型三脚架型多苯并咪唑季铵盐类化合物的合成及性能
}

\author{
张成路* 国阳陈莹孙丽杰程安琪 \\ 赵娜赵宝成唐杰袭焕 \\ (辽宁师范大学化学化工学院 辽宁省生物与制药重点实验室 大连 116029)
}

\begin{abstract}
摘要 合成了 14 个具有复合功能的三脚架型多苯并咪唑季铵盐类化合物 3a 3n, 并对其进行了结构表征. 探究了目标 化合物 3 对 $\operatorname{Cdc} 25 \mathrm{~B}$ 和 PTP1B 的抑制活性, 结果发现, 目标产物 3d、3e、3i、3j 和 3I 对 Cdc25B 表现出优良的抑制 活性, 其中 $3 \mathbf{d} 、 3 \mathbf{e} 、 3 \mathbf{j}$ 和 $3 \mathbf{I}$ 的 $\mathrm{IC}_{50}$ 值低于参照物钒酸钠, 有望成为用于治疗癌症的潜在的 $\mathrm{Cdc} 25 \mathrm{~B}$ 抑制剂; 3d、3e、 $3 \mathbf{i}$ 和 31 对 PTP1B 表现出良好的抑制活性, 31 的 $\mathrm{IC}_{50}$ 值低于参照物齐墩果酸, 有望作为用于治糖尿病潜在的 PTP1B 抑 制剂. 研究了生命体系中重要的 6 种不同阴离子对 4 个代表性目标化合物的荧光猝灭, 结果发现, I- 对目标化合物表现 出了优良的猝灭效果, 其中对 $3 \mathbf{c}$ 和 $3 \mathbf{e}$ 猝灭作用最为明显, 有望成为一种潜在的 $\mathrm{I}^{-}$苂光识别探针.
\end{abstract}

关键词 苯并咪唑; 药物活性; 苂光探针; Cdc25B; PTP1B

\section{Synthesis and Properties of Novel Tripod Type Multi-benzimidazole Quaternary Ammonium Compounds}

\author{
Zhang, Chenglu* Guo, Yang Chen, Ying Sun, Lijie Cheng, Anqi \\ Zhao, Na Zhao, Baocheng Tang, Jie Xi, Huan \\ (Liaoning Provincial Key Laboratory of Biotechnology and Drug Discovery, College of Chemistry and Chemical Engineering, \\ Liaoning Normal University, Dalian 116029)
}

\begin{abstract}
Fourteen novel tripod type multi-benzimidazole quaternary ammoniums $(\mathbf{3 a} \sim \mathbf{3 n})$ with complex functions were synthesized for the first time and the structures of the target molecules were well characterized. The inhibitory activities of Cdc25B and PTP1B of $\mathbf{3}$ were explored. The results showed that $\mathbf{3 d}, \mathbf{3 e}, \mathbf{3 i}, \mathbf{3} \mathbf{j}$ and $\mathbf{3} \mathbf{l}$ exhibit excellent inhibitory activity against $\mathrm{Cdc} 25 \mathrm{~B}$, wherein the $\mathrm{IC}_{50}$ values of $\mathbf{3 d}, \mathbf{3 e}, \mathbf{3} \mathbf{j}$ and $\mathbf{3 l}$ were lower than the reference $\mathrm{Na}_{3} \mathrm{VO}_{4}$, which were expected to be potential Cdc25B inhibitors for the treatment of cancer. Compounds 3d, 3e, 3i and $\mathbf{3 l}$ showed good inhibitory activity against PTP1B, wherein the $\mathrm{IC}_{50}$ value of $\mathbf{3 l}$ was lower than the reference oleanolic acid, which may be used as a potential PTP1B inhibitor for the treatment of diabetes. Meanwhile, the fluorescence quenching of six kinds of anions on four target compounds was also studied. It was found that $\mathrm{I}^{-}$exhibited excellent quenching effect, $\mathbf{3 c}$ and $\mathbf{3 e}$ behaved the best quenching effect and may be a potential recognition fluorescent $\mathrm{I}^{-}$sensor probe.
\end{abstract}

Keywords benzimidazole; drug activity; fluorescent sensor probe CDc25B; PTP1B

含苯并咪唑基元的分子易与酶、DNA 和 RNA 等形 成氢键, 因此在医药、农药和酶工程中具有重要的应用 价值而受到广泛的关注 ${ }^{[1,2]}$. 通过对苯并咪唑进行结构 修饰, 可获得结构多样化的衍生物而作为药物分子的药 效基团, 在抗菌 ${ }^{[\sim 7]}$ 、抗病毒 ${ }^{[8 \sim 12]}$ 、抗糖尿病 ${ }^{[13 \sim 17]}$ 和抗 癌 ${ }^{[18,19]}$ 等方面发挥重要作用, 这无疑具有重要意义.

细胞周期分裂蛋白(cell division cycle 25, Cdc25B)
属于高保守的双位点磷酸酯酶, 多数癌症的发病原因与 其过度表达相关 ${ }^{[20]}$, 因此优良的抑制剂可有效阻断癌 细胞的增殖, 抑制癌症 ${ }^{[21]}$. 蛋白酪氨酸磷酸酶 1B (protein tyrosine phosphatase 1B, PTP1B)在肿瘤中可能起类 似癌基因的作用, 它更是一种肥胖症的特异靶基因 ${ }^{[22]}$. 高活性的 PTP1B 抑制剂可降低其对胰岛素信号传导过 程的影响, 对治疗肥胖症与糖尿病具有重大意义 ${ }^{[23,24]}$.

\footnotetext{
* E-mail: zhangchenglu@Innu.edu.cn

Received December 8, 2014; revised March 25, 2015; published online April 30, 2015

Project supported by the Science and Technology Research Program of Liaoning Provincial Department of Education (No. 2009A426).

辽宁省教育厅科学技术研究(No. 2009A426)资助项目.
} 
阴离子识别在生命、环境和医学等领域中的重要作 用已引起人们极大的关注. 当阴离子结合到识别基团 时, 可通过监测受体分子的荧光信号的变化, 实现对阴 离子的传感, 发现特异阴离子识别的荧光探针, 因此研 发新型的阴离子受体已成为当今主客体化学研究的热 点 ${ }^{[25,26]}$.

三氯三乙胺具有较好的分子柔性, 易与不同的受体 结合, 以其为模板分子, 在其结构上引入能与酶活性部 位形成氢键的多个苯并咪唑，使多活性基团在同一分子 中聚集, 可能改善分子生物活性. 若活性组分以阳离子 形式存在, 可直接透过细胞膜, 可增加与受体的亲合力 和选择性, 发现活性更强的新型 PTP1B 和 Cde25B 的抑 制剂, 而且许多季铵盐分子因具有高效的杀菌和低毒活 性 ${ }^{[27]}$ 而被广泛关注.

为了发现和笁选较好的 Cdc25B、PTP1B 抑制剂和 阴离子荧光识别探针, 我们将苯并咪唑结构中的两个 $\mathrm{N}$ 原子同时修饰, 以获得药效基团片段中 $(\mathrm{N}-\mathrm{CH}=\mathrm{N})$ 高活 性的氢原子, 利用结构中的多个杂原子、活泼氢和三脚 架型空间结构期望实现对阴离子的专一性识别, 目标产 物的合成方法如 Scheme 1 所示.

\section{1 结果与讨论}

\section{1 目标化合物 3 的合成与表征}

将中间体 1 在强碱的作用下与 3 个苯并咪唑对接合 成了重要的中间体 2.2 分别与不同取代芳香苄氯季铵化 合成目标产物 3a 3n. 通过 IR, ${ }^{1} \mathrm{H}$ NMR 和 HRMS 等 对目标分子进行了结构表征. 在 IR 谱图中, 3 在 3300 $\mathrm{cm}^{-1}$ 左右未见苯并咪唑中的 $\mathrm{N}-\mathrm{H}$ 的伸缩振动峰, 在 1650 $1500 \mathrm{~cm}^{-1}$ 范围内出现了 $\mathrm{C}=\mathrm{N}$ 和苯环上 $\mathrm{C}=\mathrm{C}$ 的 吸收峰; ${ }^{1} \mathrm{H}$ NMR 谱图中, 在 $\delta 9.80$ 附近出现了苯并咪唑 $\mathrm{C}(2)-\mathrm{H}$ 的质子峰处并且在 $721 \sim 747 \mathrm{~cm}^{-1}$ 之间; HRMS 谱图中, 所有的化合物均出现了 $\left[\mathrm{M}-3 \mathrm{BF}_{4}^{-}\right]^{+} 、[\mathrm{M}-$ $\left.3 \mathrm{BF}_{4}^{-}\right]^{2+} 、\left[\mathrm{M}-3 \mathrm{BF}_{4}^{-}\right]^{3+}$ 的峰, 说明目标化合物已成功 合成.

\section{2 实验条件的优化}

\subsection{1中间体 2 合成条件的选择}

中间体 2 的合成已有文献报道 ${ }^{[28]}$, 但其合成的产率 仅为 $52 \%$. 为了提高其合成收率, 我们探究了碱性、溶 剂、反应的温度和物料比对反应的影响, 实验结果由文 献中 $52 \%$ 的收率提高到了 $88 \%$, 结果如表 1 .

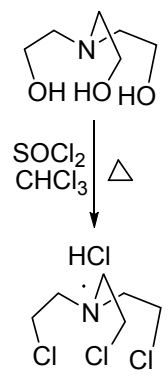

1

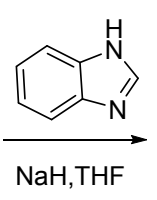

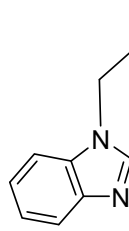

$-N$

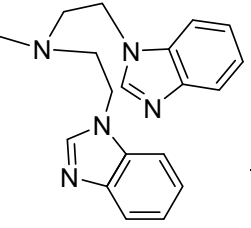

2

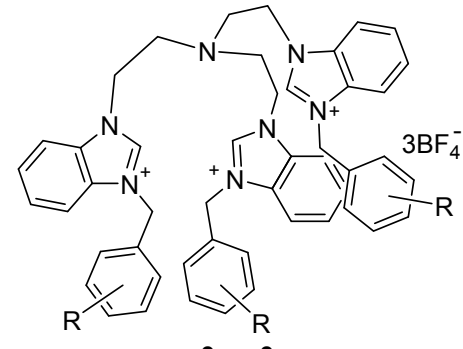

$3 a \sim 3 n$

$\mathrm{Ar}=\mathrm{C}_{6} \mathrm{H}_{5}(\mathbf{a}), 4-\mathrm{MeC}_{6} \mathrm{H}_{4}(\mathbf{b}), 4-\mathrm{MeOC}_{6} \mathrm{H}_{4}(\mathbf{c}), 4-\mathrm{NH}_{2} \mathrm{C}_{6} \mathrm{H}_{4}(\mathbf{d}), 3-\mathrm{NH}_{2} \mathrm{C}_{6} \mathrm{H}_{4}(\mathbf{e}), 4-\mathrm{NO}_{2} \mathrm{C}_{6} \mathrm{H}_{4}(\mathbf{f}), 4-\mathrm{ClC}_{6} \mathrm{H}_{4}(\mathbf{g}), 4-\mathrm{BrC}_{6} \mathrm{H}_{4}(\mathbf{h})$, $\mathrm{X}=\mathrm{Cl}, \mathrm{Br}$ $4-\mathrm{FC}_{6} \mathrm{H}_{4}(\mathbf{i}), 3-\left(1-\mathrm{C}_{6} \mathrm{H}_{5} \mathrm{SO}_{2}\right) \mathrm{C}_{8} \mathrm{H}_{6} \mathrm{~N}(\mathbf{j}), 2-\mathrm{C}_{4} \mathrm{H}_{3} \mathrm{~S}(\mathbf{k}), 2-\mathrm{C}_{5} \mathrm{H}_{4} \mathrm{~N}(\mathrm{I}), n-\mathrm{C}_{6} \mathrm{H}_{13}(\mathbf{m}), n-\mathrm{C}_{8} \mathrm{H}_{1}(\mathbf{n})$

图式 1 化合物 $3 a \sim 3 n$ 的合成

Scheme 1 Synthesis of compounds $\mathbf{3 a} \sim \mathbf{3 n}$

表 1 中间体 2 合成条件的优化

Table 1 Synthesis of intermediate $\mathbf{2}$ in optimal conditions

\begin{tabular}{|c|c|c|c|c|c|c|c|c|c|c|c|}
\hline Entry & Base & Solvent & Temp. $/{ }^{\circ} \mathrm{C}$ & $\begin{array}{c}\text { Material } \\
\text { ratio }\end{array}$ & Yield/\% & Entry & Base & Solvent & Temp. $/{ }^{\circ} \mathrm{C}$ & $\begin{array}{l}\text { Material } \\
\text { ratio }\end{array}$ & Yield/\% \\
\hline 1 & $\mathrm{Na}_{2} \mathrm{CO}_{3}$ & $\mathrm{CH}_{3} \mathrm{CN}$ & 60 & $1: 3.1$ & 32 & 8 & $\mathrm{NaH}$ & THF & 25 & $1: 3.1$ & 30 \\
\hline 2 & $\mathrm{NaOH}$ & $\mathrm{CH}_{3} \mathrm{CN}$ & 60 & $1: 3.1$ & 55 & 9 & $\mathrm{NaH}$ & THF & 40 & $1: 3.1$ & 48 \\
\hline 3 & $\mathrm{NaH}$ & $\mathrm{CH}_{3} \mathrm{CN}$ & 60 & $1: 3.1$ & 63 & 10 & $\mathrm{NaH}$ & THF & 55 & $1: 3.1$ & 63 \\
\hline 4 & $\mathrm{Na}_{2} \mathrm{CO}_{3} / \mathrm{NaOH}$ & $\mathrm{CH}_{3} \mathrm{CN}$ & 60 & $1: 3.1$ & 49 & 11 & $\mathrm{NaH}$ & THF & 70 & $1: 3.1$ & 87 \\
\hline 5 & $\mathrm{NaH}$ & $\mathrm{C}_{2} \mathrm{H}_{5} \mathrm{OH}$ & 60 & $1: 3.1$ & 52 & 12 & $\mathrm{NaH}$ & THF & 70 & $1: 2.8$ & 65 \\
\hline 6 & $\mathrm{NaH}$ & THF & 60 & $1: 3.1$ & 84 & 13 & $\mathrm{NaH}$ & THF & 70 & $1: 3.0$ & 72 \\
\hline \multirow[t]{2}{*}{7} & $\mathrm{NaH}$ & $\mathrm{CH}_{3} \mathrm{COCH}_{3}$ & 60 & $1: 3.1$ & 42 & 14 & $\mathrm{NaH}$ & THF & 70 & $1: 3.2$ & 88 \\
\hline & & & & & & 15 & $\mathrm{NaH}$ & THF & 70 & $1: 3.4$ & 84 \\
\hline
\end{tabular}


实验表明，中间体 2 合成的最佳条件为: 物料比为 三氯三乙胺 : 苯并咪唑 $(\mathrm{mol} / \mathrm{mol})=1: 3.2, \mathrm{THF}$ 作溶剂, $\mathrm{NaH}$ 作碱性条件, 反应温度为 $70{ }^{\circ} \mathrm{C}$, 反应时间 $4 \mathrm{~h}$, 中 间体 2 可以达到 $88 \%$ 的收率.

\subsection{2 目标化合物 3 合成条件的选择}

有关苯并咪坐季铵盐合成中涉及的溶剂种类较多, 温度的变化较广, 反应的时间也各有不同, 尤其是对于 含有 3 个苯并咪唑结构的季铵盐合成鲜见报道. 为了以 较高收率完成目标产物的合成, 我们以中间体 $\mathbf{2}$ 与苄氯 的反应为模板反应，探究了反应时间、温度和溶剂的影 响, 结果如表 2 .

实验结果表明, 合成目标化合物的最佳条件为: 反 应温度 $110{ }^{\circ} \mathrm{C}, \mathrm{DMF}$ 为溶剂, 反应时间为 $48 \mathrm{~h}$, 目标 产物的最高收率可达 $78 \%$.

\section{$1.3 \mathrm{Cdc} 25 \mathrm{~B}$ 和 PTP1B 的抑制活性测试}

对目标化合物 $3 \mathrm{a} \sim 3 \mathrm{n}$ 初篮选择的浓度均为 5 $\mu \mathrm{g} / \mathrm{mL}, \mathrm{Na}_{3} \mathrm{VO}_{4}$ 是酪氨酸磷酸酯酶广谱的抑制剂, 可作 为 $\mathrm{Cdc} 25 \mathrm{~B}$ 抑制活性的对照药物, 齐墩果酸(Oleanolic acid)是通过 PTP1B 靶点笁选出来的阳性抑制剂, 可作 为 PTP1B 的抑制活性的对照药物 ${ }^{[29]}$ ，对于抑制率 $>50 \%$ 的样品, 再测试活性剂量依赖关系, 即 $\mathrm{IC}_{50}$ 值. 具体抑 制活性笁选实验方法: 按照参考文献[30, 31]的方法进 行. 本实验由国家新药中心协助完成, 结果如表 3 所示.

\section{4 目标产物的阴离子识别}

\subsection{1 不同阴离子对目标化合物荧光数据图}

以乙腈与水 $[V$ (乙腈) $: V($ 水 $)=99: 1]$ 为溶剂, 配制
浓度为 $2 \times 10^{-4} \mathrm{~mol} / \mathrm{L}$ 、分别含人体内常见的 6 种阴离子 $\mathrm{F}^{-} 、 \mathrm{Cl}^{-} 、 \mathrm{Br}^{-} 、 \mathrm{I}^{-} 、 \mathrm{H}_{2} \mathrm{PO}_{4}^{-}$和 $\mathrm{CH}_{3} \mathrm{COO}^{-}$的溶液 $\mathrm{A}$ 备用. 将 $3 \mathbf{h} 、 3 \mathbf{j} 、 3 \mathbf{k}$ 和 $3 \mathbf{l}$ 分别溶于乙腈和水 $[V$ (乙腈) : $V($ 水 $)=99: 1]$ 中, 配制浓度为 $1 \times 10^{-5} \mathrm{~mol} / \mathrm{L}$ 的溶液 $\mathrm{B}$ 备用. 将 A、B 两种溶液等体积比混合后进行苂光性能 测试，具体实验方法：按照参考文献[32，33]的方法进 行. 测试结果如图 1 所示.

\section{4 .2 不同阴离子对目标化合物荧光的猝灭率}

分析上述荧光谱图可发现， $\mathrm{I}^{-}$离子对 4 种代表性目 标化合物均表现出优良的猝灭效果. 根据荧光测试结 果, 通过公式: 淬灭率 $=I_{0}-I / I_{0}\left(I_{0}\right.$ 为配体溶液的最大苂 光强度; $I$ 为碘离子后溶液的最大荧光强度), 可以计算 出 $\mathrm{I}^{-}$离子对目标化合物 $3 \mathbf{h} 、 3 \mathbf{j} 、 3 \mathbf{k}$ 和 $3 \mathrm{l}$ 苂光的淬灭 率, 结果如图 2 所示.

\section{2 结论}

通过简捷高效的方法合成了 14 个含有苯并咪唑结 构的新型三脚架型季铵盐类化合物. 探究了中间体 $\mathbf{2}$ 和 目标分子 3 的最佳合成条件，并对其进行了结构的表征 和药物活性的检测. 研究发现, 目标 $3 \mathbf{d} 、 3 \mathbf{3} 、 3 \mathbf{3} 、 3 \mathbf{j}$ 和 31 对 $\mathrm{Cdc} 25 \mathrm{~B}$ 表现出良好的抑制活性，具有较低的 $\mathrm{IC}_{50}$ 值, 其中 $3 \mathrm{e}$ 的效果最为明显 $\mathrm{IC}_{50}=(0.65 \pm 0.12) \mu \mathrm{g} / \mathrm{mL}$. 构效关系表明, 苯环上含有氨基、氟离子及引入杂环时, 该类化合物对 $\mathrm{Cdc} 25 \mathrm{~B}$ 的抑制具有较好的提升作用， 3d、3e、3i、3j 和 31 有望成为用于治疗癌症的潜在的 Cdc25B 抑制剂; 3d、3e、3i 和 31 对 PTP1B 表现出良好

表 2 目标产物 3 的合成条件的优化

Table 2 Synthesis of the target product 3 in optimal conditions

\begin{tabular}{ccccc||ccccc||ccccc}
\hline Entry & Solvent & Temp. $/{ }^{\circ} \mathrm{C}$ & Time/h & Yield\% & Entry & Solvent & Temp. $/{ }^{\circ} \mathrm{C}$ & Time $/ \mathrm{h}$ & Yield\% & Entry & Solvent & Temp. $/{ }^{\circ} \mathrm{C}$ & Time $/ \mathrm{h}$ & Yield\% \\
\hline 1 & DMF & 80 & 40 & 48 & 6 & DMF & 110 & 12 & 25 & 11 & DMF & 120 & 40 & 70 \\
2 & DMF & 83 & 40 & 54 & 7 & DMF & 110 & 24 & 48 & 12 & $\mathrm{Ph}_{-} \mathrm{CH}_{3}$ & 83 & 40 & 32 \\
3 & DMF & 90 & 40 & 59 & 8 & DMF & 110 & 36 & 60 & 13 & $\mathrm{DMSO}$ & 83 & 40 & 41 \\
4 & DMF & 100 & 40 & 63 & 9 & DMF & 110 & 48 & 78 & 14 & $\mathrm{CH}_{3} \mathrm{CN}$ & 83 & 40 & 35 \\
5 & DMF & 110 & 40 & 75 & 10 & DMF & 110 & 60 & 59 & & & & \\
\hline
\end{tabular}

表 3 目标化合物对 Cdc25B 和 PTP1B 的抑制率

Table 3 Inhibition rate of the target compounds for Cdc25B and PTP1B

\begin{tabular}{|c|c|c|c|c|c|c|c|c|c|}
\hline \multirow{2}{*}{ Compd. } & \multirow{2}{*}{$\mathrm{Ar}$} & \multirow{2}{*}{$\mathrm{X}$} & \multicolumn{2}{|c|}{$\mathrm{IC}_{50}{ }^{a} /\left(\mu \mathrm{mol} \cdot \mathrm{L}^{-1}\right)$} & \multirow{2}{*}{ Compd. } & \multirow{2}{*}{$\mathrm{Ar}$} & \multirow{2}{*}{$X$} & \multicolumn{2}{|c|}{$\mathrm{IC}_{50}{ }^{a} /\left(\mu \mathrm{mol} \cdot \mathrm{L}^{-1}\right)$} \\
\hline & & & Cdc25B & РTP1B & & & & $\mathrm{Cdc} 25 \mathrm{~B}$ & РTP1B \\
\hline $3 a$ & $\mathrm{C}_{6} \mathrm{H}_{5}$ & $\mathrm{Cl}$ & NA & NA & $3 \mathbf{h}$ & $4-\mathrm{BrC}_{6} \mathrm{H}_{4}$ & $\mathrm{Cl}$ & NA & NA \\
\hline $3 \mathbf{b}$ & 4- $\mathrm{CH}_{3} \mathrm{C}_{6} \mathrm{H}_{4}$ & $\mathrm{Cl}$ & NA & NA & $3 \mathbf{i}$ & $4-\mathrm{FC}_{6} \mathrm{H}_{4}$ & $\mathrm{Cl}$ & $2.85 \pm 0.69$ & $3.54 \pm 0.54$ \\
\hline $3 c$ & $4-\mathrm{CH}_{3} \mathrm{OC}_{6} \mathrm{H}_{4}$ & $\mathrm{Cl}$ & NA & NA & $3 \mathbf{j}$ & $3-\left(1-\mathrm{C}_{6} \mathrm{H}_{5} \mathrm{SO}_{2}\right) \mathrm{C}_{8} \mathrm{H}_{6} \mathrm{~N}$ & $\mathrm{Br}$ & $0.86 \pm 0.1$ & NA \\
\hline 3d & 4- $\mathrm{NH}_{2} \mathrm{C}_{6} \mathrm{H}_{4}$ & $\mathrm{Cl}$ & $1.27 \pm 0.13$ & $1.90 \pm 0.50$ & $3 \mathbf{k}$ & $2-\mathrm{C}_{4} \mathrm{H}_{3} \mathrm{~S}$ & $\mathrm{Cl}$ & NA & NA \\
\hline $3 e$ & $3-\mathrm{NH}_{2} \mathrm{C}_{6} \mathrm{H}_{4}$ & $\mathrm{Cl}$ & $0.65 \pm 0.12$ & $2.03 \pm 0.56$ & 31 & $2-\mathrm{C}_{5} \mathrm{H}_{4} \mathrm{~N}$ & $\mathrm{Cl}$ & $0.91 \pm 0.34$ & $0.31 \pm 0.05$ \\
\hline $3 f$ & $4-\mathrm{NO}_{2} \mathrm{C}_{6} \mathrm{H}_{4}$ & $\mathrm{Cl}$ & NA & NA & $3 \mathrm{~m}$ & $\mathrm{C}_{6} \mathrm{H}_{13}$ & $\mathrm{Br}$ & NA & NA \\
\hline $3 g$ & $4-\mathrm{ClC}_{6} \mathrm{H}_{4}$ & $\mathrm{Cl}$ & NA & NA & $3 n$ & $\mathrm{C}_{8} \mathrm{H}_{17}$ & $\mathrm{Br}$ & NA & NA \\
\hline $\mathrm{Na}_{3} \mathrm{VO}_{4}^{b}$ & - & 一 & $1.86 \pm 0.24$ & - & Oleanolic acid ${ }^{c}$ & - & - & - & $1.11 \pm 0.09$ \\
\hline
\end{tabular}

${ }^{a}$ Values tested at $5 \mu \mathrm{g} / \mathrm{mL}$ concentration. ${ }^{b} \mathrm{Na}_{3} \mathrm{VO}_{4}$ as a positive control. ${ }^{c}$ Oleanolic acid as a positive control. 

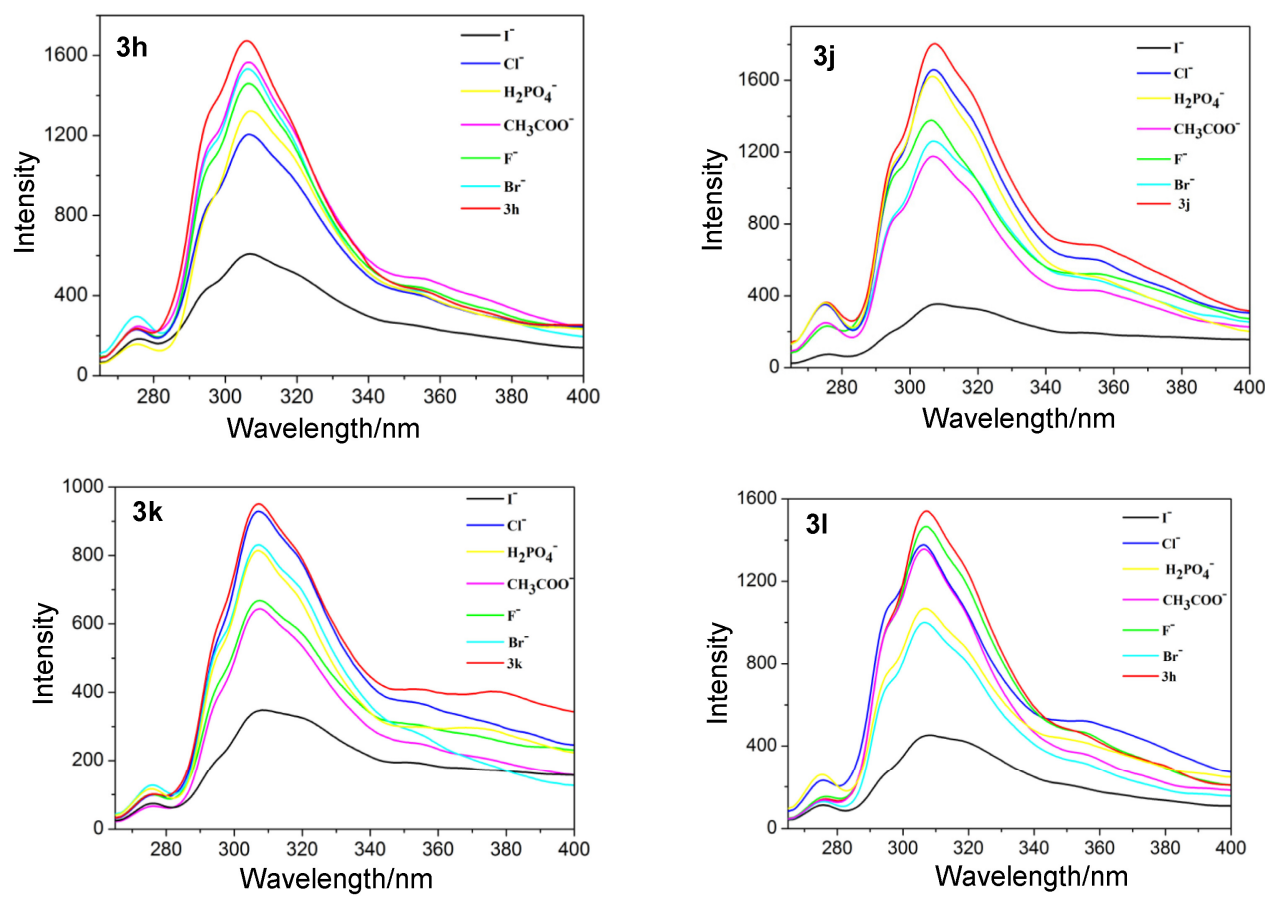

图 1 化合物 $3 \mathrm{~h} 、 3 \mathrm{j} 、 3 \mathrm{k}$ 和 $3 \mathrm{I}$ 随不同阴离子的苂光变化

Figure 1 Fluorescence change of $\mathbf{3 h}, \mathbf{3} \mathbf{j}, \mathbf{3 k}$ and $\mathbf{3 l}$ with different anions
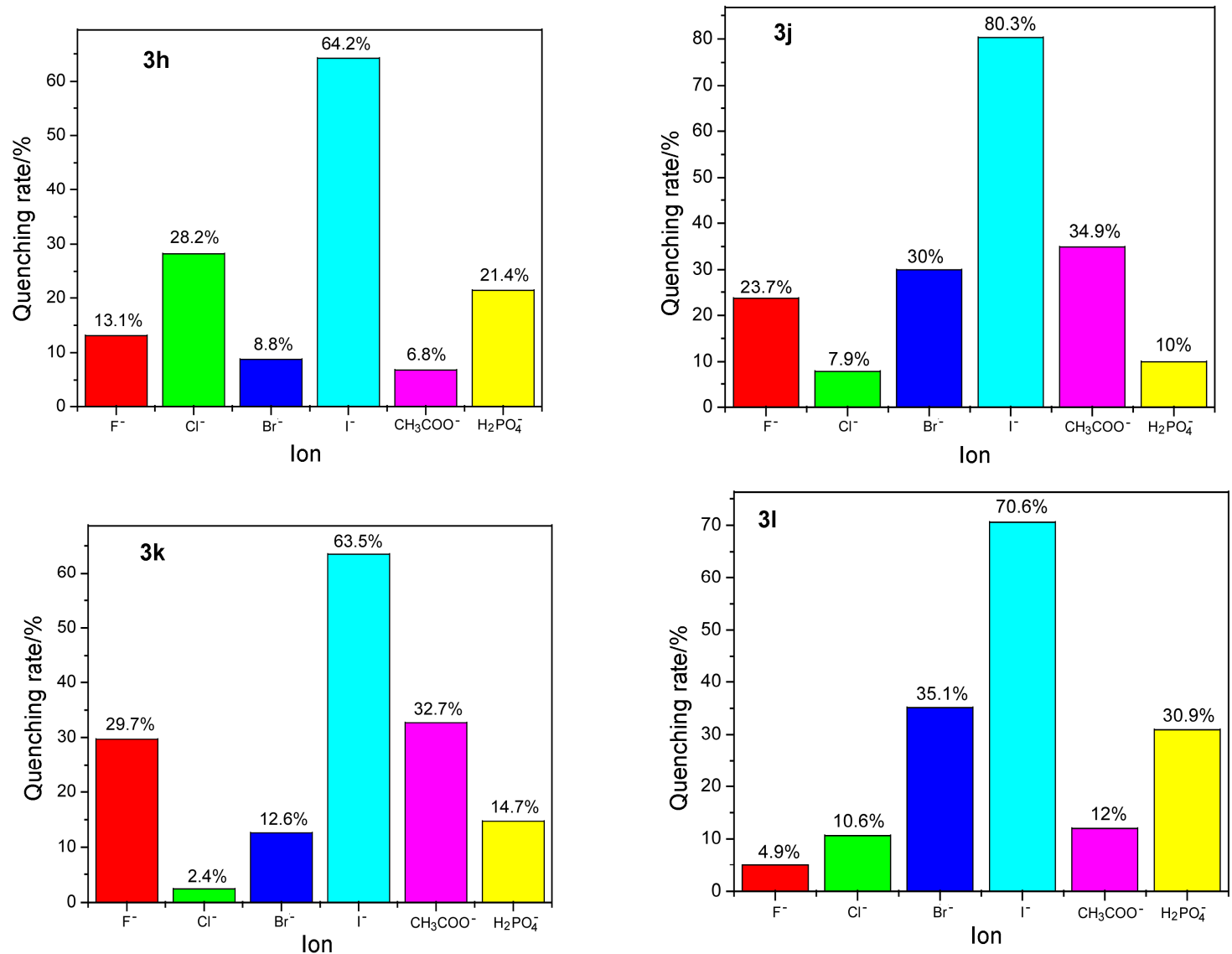

图 2 化合物 $3 \mathbf{h} 、 3 \mathbf{j} 、 3 \mathbf{k}$ 和 $3 \mathbf{l}$ 随不同阴离子的苂光猝灭率

Figure 2 Compound $\mathbf{3 h} 、 \mathbf{3} \mathbf{j} 、 \mathbf{3 k}$ and $\mathbf{3 l}$ fluorescence quenching with different anions

的抑制活性, 31 的效果最为明显 $\mathrm{IC}_{50}=(0.31 \pm 0.05)$

$\mu \mathrm{g} / \mathrm{mL}$ ，说明吡啶基团的引入促进了 PTP1B 的抑制活 
性, 31 可作为用于治糖尿病的潜在的 PTP1B 抑制剂. 通 过苂光光谱和猝灭率计算结果发现, 新型三脚架型苯并 咪唑季铵盐类化合物对 $\mathrm{I}^{-}$具有优异的苂光识别作用, $\mathrm{I}^{-}$ 对所有化合物都具有猝灭作用，当形成季铵盐的芳香基 才上的取代基为吲哚基团和吡啶基团的时候可以看出 其猝灭的趋势明显, 其中 $\mathrm{I}^{-}$对目标化合物 $\mathbf{3} \mathbf{j}$ 猝灭作用最 为明显, 猝灭率可以达到 $80.3 \%$, 有望成为 $\mathrm{I}^{-}$荧光识别 探针.

\section{3 实验部分}

\section{1 仪器与试剂}

AVANCE $500 \mathrm{MHz}$ NMR 核磁共振波谱仪; Accurate-Mass TOF LC/MS(精确质量液相色谱飞行时间质谱 联用仪)高分辨质谱仪; TENSOR27 傅立叶变换红外光 谱仪; X-5 型数字显微熔点测定仪(温度计未经校正); WFH-203B 型三用紫外分析仪. 所用的试剂均为市售的 分析纯, 两个测试蛋白 PTP1B、Cdc25B 均由国家新药 笁选中心实验室利用大肠杆菌表达并纯化得到的, 纯度 在 $90 \%$ 以上. 取代茮卤根据参考文献[34 42] 由实验室 自行合成.

\section{2 实验方法}

化合物三氯三乙胺(1)参考文献[43]合成, 收率为 $85 \%$, m.p. $210.4 \sim 212.1{ }^{\circ} \mathrm{C}$, 与文献值相符.

3.2 .1 化合物三(2-(1H-苯并咪唑基)乙基)胺(2)的合 成新方法

在 $\mathrm{N}_{2}$ 作为保护气体下, 将 $10 \mathrm{mmol}$ 苯并咪唑加入 到三口瓶中, 并加入 $20 \mathrm{~mL}$ 无水的四氢呋喃, 搅拌使其 充分的溶解, 再缓慢分批地加入 $12 \mathrm{mmol}$ 的 $\mathrm{NaH}$, 保持 在室温下搅拌 $30 \mathrm{~min}$, 再缓慢地加入 $3.1 \mathrm{mmol}$ 的三氯三 乙胺盐酸盐 1 , 加入完毕后, 在回流的条件下, 反应 $4 \mathrm{~h}$, 反应结束后加入 $40 \mathrm{~mL}$ 的蒸馏水, 淡黄色固体产生, 抽 滤得粗产品, 粗产品通过柱层析分离提纯 $[V($ 乙 醇) : $V($ 乙酸乙酯 $)=1: 1]$, 得到白色的产品 2 , 产率约 为 $88 \%$, m.p. $176.5 \sim 177.9{ }^{\circ} \mathrm{C}$, 与文献值相符.

3.2 .2 三-[2-(1H-苯并咪唑基)乙基]胺氟喼酸盐 $(3 \mathbf{a} \sim$ $3 n)$ 的合成通法

在 $\mathrm{N}_{2}$ 作为保护气体下, 将 $1 \mathrm{mmol} 2$ 加到 $100 \mathrm{~mL}$ 三

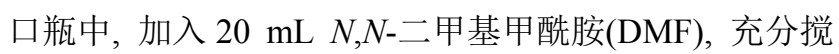
拌使其溶解, 再分批加入 $3.2 \mathrm{mmol}$ 的芐氯(或苠溴), 在 $110{ }^{\circ} \mathrm{C}$ 的条件下反应 $48 \mathrm{~h}$, 将体系冷却至室温, 减压蒸 去溶剂, 得到灰白色的固体, 将固体溶解在 $20 \mathrm{~mL}$ 的乙 醇溶液中, 加入 $3 \mathrm{mmol}$ 的 $\mathrm{NH}_{4} \mathrm{BF}_{4}$, 有白色固体析出, 减压过滤, 固体用乙醇进行快速柱层析, 得到白色的固 体.
三-[2-(3-茮基- $1 H$-苯并咪唑-2-亚基)乙基]胺氟硼酸 盐(3a)：白色固体，收率 78.2\%. m.p. 185.5 186.7 ${ }^{\circ} \mathrm{C}$; ${ }^{1} \mathrm{H}$ NMR (500 MHz, DMSO-d $) \delta: 9.79$ (s, $\left.3 \times 1 \mathrm{H}\right), 7.95$ $(\mathrm{d}, J=8.15 \mathrm{~Hz}, 3 \times 1 \mathrm{H}), 7.94(\mathrm{~d}, J=8.10 \mathrm{~Hz}, 3 \times 1 \mathrm{H})$, $7.67 \sim 7.61(\mathrm{~m}, 3 \times 2 \mathrm{H}), 7.48(\mathrm{~d}, J=7.25 \mathrm{~Hz}, 3 \times 2 \mathrm{H}), 7.34$ $(\mathrm{d}, J=4.8 \mathrm{~Hz}, 3 \times 2 \mathrm{H}), 5.74(\mathrm{~s}, 3 \times 2 \mathrm{H}), 4.53(\mathrm{t}, J=6.65$ $\mathrm{Hz}, 3 \times 2 \mathrm{H}), 3.30(\mathrm{t}, J=6.65 \mathrm{~Hz}, 3 \times 2 \mathrm{H})$; IR $(\mathrm{KBr}) v$ : $3047(\mathrm{C}-\mathrm{H}), 2938(\mathrm{C}-\mathrm{H}), 1634(\mathrm{C}=\mathrm{N}), 1590(\mathrm{ArC}=$ C), $1072(\mathrm{C}-\mathrm{N}), 732(\mathrm{C}-\mathrm{H}) \mathrm{cm}^{-1}$. HRMS (ESI) calcd for $\mathrm{C}_{48} \mathrm{H}_{48} \mathrm{~B}_{2} \mathrm{~F}_{8} \mathrm{~N}_{7}\left[\mathrm{M}-\mathrm{BF}_{4}^{-}\right]^{+}$896.4030, found 896.4023; $\mathrm{C}_{48} \mathrm{H}_{48} \mathrm{BF}_{4} \mathrm{~N}_{7}\left[\mathrm{M}-2 \mathrm{BF}_{4}\right]^{2+} 404.7000$, found 404.6998; $\mathrm{C}_{48} \mathrm{H}_{48} \mathrm{~N}_{7}\left[\mathrm{M}-3 \mathrm{BF}_{4}\right]^{3+} 240.7990$, found 240.7998 .

三-[2-(3-对甲基苄基- $1 H$-苯并咪唑-2-亚基)乙基]胺 氟硼酸盐(3b): 白色固体，收率 79.6\%. m.p. 198.3 $199.9{ }^{\circ} \mathrm{C} ;{ }^{1} \mathrm{H}$ NMR (500 MHz, DMSO- $\left.d_{6}\right) \delta: 9.89$ (s, $3 \times$ $1 \mathrm{H}), 7.94(\mathrm{~d}, J=8.15 \mathrm{~Hz}, 3 \times 1 \mathrm{H}), 7.91(\mathrm{~d}, J=8.10 \mathrm{~Hz}$, $3 \times 1 \mathrm{H}), 7.66 \sim 7.61(\mathrm{~m}, 3 \times 2 \mathrm{H}), 7.40(\mathrm{~d}, J=8.05 \mathrm{~Hz}, 3 \times$ $2 \mathrm{H}), 7.18(\mathrm{~d}, J=8.0 \mathrm{~Hz}, 3 \times 2 \mathrm{H}), 5.68(\mathrm{~s}, 3 \times 2 \mathrm{H}), 4.54(\mathrm{t}$, $J=6.65 \mathrm{~Hz}, 3 \times 2 \mathrm{H}), 3.30(\mathrm{t}, J=6.65 \mathrm{~Hz}, 3 \times 2 \mathrm{H}), 2.22(\mathrm{~s}$, $3 \times 3 \mathrm{H})$; IR $(\mathrm{KBr}) v$ : $3048(\mathrm{C}-\mathrm{H}), 2922(\mathrm{C}-\mathrm{H}), 1648$ $(\mathrm{C}=\mathrm{N}), 1532(\mathrm{Ar}, \mathrm{C}=\mathrm{C}), 1032(\mathrm{C}-\mathrm{N}), 744(\mathrm{C}-\mathrm{H})$ $\mathrm{cm}^{-1}$. HRMS (ESI) calcd for $\mathrm{C}_{51} \mathrm{H}_{54} \mathrm{~B}_{2} \mathrm{~F}_{8} \mathrm{~N}_{7}\left[\mathrm{M}-\mathrm{BF}_{4}^{-}\right]^{+}$ 938.4499, found 938.4497; $\mathrm{C}_{51} \mathrm{H}_{54} \mathrm{BF}_{4} \mathrm{~N}_{7}\left[\mathrm{M}-2 \mathrm{BF}_{4}\right]^{2+}$ 425.7235, found 425.7232; $\mathrm{C}_{51} \mathrm{H}_{54} \mathrm{~N}_{7}\left[\mathrm{M}-3 \mathrm{BF}_{4}\right]^{3+}$ 254.8147 , found 254.8131 .

三-[2-(3-对甲基氧苄基- $1 H$-苯并咪唑-2-亚基)乙基] 胺氟硼酸盐 $(3 \mathrm{c})$ : 白色固体，收率 78.5\%. m.p. 180.9 $181.7{ }^{\circ} \mathrm{C} ;{ }^{1} \mathrm{H}$ NMR (500 MHz, DMSO- $\left.d_{6}\right) \delta: 9.70$ (s, $3 \times$ $1 \mathrm{H}), 7.99$ (d, $J=8.05 \mathrm{~Hz}, 3 \times 1 \mathrm{H}), 7.92(\mathrm{~d}, J=8.0 \mathrm{~Hz}, 3 \times$ $1 \mathrm{H}, 7.68 \sim 7.61(\mathrm{~m}, 3 \times 2 \mathrm{H}), 7.46(\mathrm{t}, J=8.65 \mathrm{~Hz}, 3 \times 2 \mathrm{H})$, $6.94(\mathrm{~d}, J=8.65 \mathrm{~Hz}, 3 \times 2 \mathrm{H}), 5.64(\mathrm{~s}, 3 \times 2 \mathrm{H}), 4.50(\mathrm{t}, J=$ $6.55 \mathrm{~Hz}, 3 \times 2 \mathrm{H}), 3.70(\mathrm{~s}, 3 \times 3 \mathrm{H}), 3.27(\mathrm{t}, J=6.5 \mathrm{~Hz}, 3 \times$ 2H); IR (KBr) v: $3055(\mathrm{C}-\mathrm{H}), 2932(\mathrm{C}-\mathrm{H}), 1662(\mathrm{C}=$ $\mathrm{N}), 1587(\mathrm{Ar}, \mathrm{C}=\mathrm{C}), 1221(\mathrm{C}-\mathrm{O}), 1083(\mathrm{C}-\mathrm{N}), 744$ $(\mathrm{C}-\mathrm{H}) \mathrm{cm}^{-1}$. HRMS (ESI) calcd for $\mathrm{C}_{51} \mathrm{H}_{54} \mathrm{~B}_{2} \mathrm{~F}_{8} \mathrm{~N}_{7} \mathrm{O}_{3}$ $\left[\mathrm{M}-\mathrm{BF}_{4}^{-}\right]^{+}$986.4347, found 986.4341; $\mathrm{C}_{51} \mathrm{H}_{54} \mathrm{BF}_{4} \mathrm{~N}_{7} \mathrm{O}_{3}$ $\left[\mathrm{M}-2 \mathrm{BF}_{4}\right]^{2+} 449.7158$, found 449.7153; $\mathrm{C}_{51} \mathrm{H}_{54} \mathrm{~N}_{7} \mathrm{O}_{3}$ $\left[\mathrm{M}-3 \mathrm{BF}_{4}\right]^{3+} 270.8096$, found 270.8098 .

三-[2-(3-对氨基苄基- $1 H$-苯并咪唑-2-亚基)乙基]胺 氟硼酸盐(3d): 白色固体，收率 78.9\%. m.p. 197.9 $198.7{ }^{\circ} \mathrm{C} ;{ }^{1} \mathrm{H}$ NMR (500 MHz, DMSO-d $\left.d_{6}\right) \delta$ : 9.85 (s, $3 \times$ $1 \mathrm{H}), 7.98(\mathrm{~d}, 3 \times 1 \mathrm{H}), 7.95(\mathrm{~d}, 3 \times 1 \mathrm{H}), 7.70 \sim 7.65(\mathrm{~m}, 3 \times$ $2 \mathrm{H}), 7.59(\mathrm{t}, J=7.3 \mathrm{~Hz}, 3 \times 2 \mathrm{H}), 7.27(\mathrm{t}, J=7.1 \mathrm{~Hz}, 3 \times$ $2 \mathrm{H}), 6.58(\mathrm{~s}, 3 \times 2 \mathrm{H}), 5.78(\mathrm{~s}, 3 \times 2 \mathrm{H}), 4.58(\mathrm{t}, J=6.25 \mathrm{~Hz}$, $3 \times 2 \mathrm{H}), 3.31(\mathrm{t}, J=6.25 \mathrm{~Hz}, 3 \times 2 \mathrm{H})$; IR $(\mathrm{KBr}) v: 3331$ 
$(\mathrm{N}-\mathrm{H}), 3189(\mathrm{C}-\mathrm{H}), 2932(\mathrm{C}-\mathrm{H}), 1654(\mathrm{C}=\mathrm{N}), 1569$ $(\mathrm{ArC}=\mathrm{C}), 1193(\mathrm{C}-\mathrm{N}), 735(\mathrm{C}-\mathrm{H}) \mathrm{cm}^{-1}$. HRMS (ESI) calcd for $\mathrm{C}_{48} \mathrm{H}_{51} \mathrm{~B}_{2} \mathrm{~F}_{8} \mathrm{~N}_{10}\left[\mathrm{M}-\mathrm{BF}_{4}^{-}\right]^{+}$941.4357, found 941.4376; $\mathrm{C}_{48} \mathrm{H}_{51} \mathrm{BF}_{4} \mathrm{~N}_{10}\left[\mathrm{M}-2 \mathrm{BF}_{4}\right]^{2+}$ 427.2163, found 427.2173; $\mathrm{C}_{48} \mathrm{H}_{51} \mathrm{~N}_{10}\left[\mathrm{M}-3 \mathrm{BF}_{4}^{-}\right]^{3+} 255.8099$, found 255.8097.

三-[2-(3-间氨基苄基- $1 H$-苯并咪唑-2-亚基)乙基]胺 氟硼酸盐(3e): 白色固体, 收率 76.4\%. m.p. 201.3 $202.6{ }^{\circ} \mathrm{C} ;{ }^{1} \mathrm{H}$ NMR $\left(500 \mathrm{MHz}, \mathrm{DMSO}-d_{6}\right) \delta: 9.69$ (s, $3 \times$ $1 \mathrm{H}), 8.03(\mathrm{t}, J=8.2 \mathrm{~Hz}, 3 \times 1 \mathrm{H}), 7.90(\mathrm{~d}, J=8.25 \mathrm{~Hz}, 3 \times$ $1 \mathrm{H}), 7.69 \sim 7.61(\mathrm{~m}, 3 \times 2 \mathrm{H}), 7.18(\mathrm{~d}, J=1.7 \mathrm{~Hz}, 3 \times 1 \mathrm{H})$, 7.03 (q, $J=8.25 \mathrm{~Hz}, 3 \times 2 \mathrm{H}), 6.92(\mathrm{q}, J=8.3 \mathrm{~Hz}, 3 \times 1 \mathrm{H})$, $6.47(\mathrm{~s}, 3 \times 2 \mathrm{H}), 5.61(\mathrm{~s}, 3 \times 2 \mathrm{H}), 4.48(\mathrm{t}, J=6.45,3 \times 2 \mathrm{H})$, 3.29 (d, $J=2.45,3 \times 2 \mathrm{H}), 3.26$ (t, $J=6.45 \mathrm{~Hz}, 3 \times 2 \mathrm{H})$; IR (KBr) v: $3343(\mathrm{~N}-\mathrm{H}), 3181(\mathrm{C}-\mathrm{H}), 2835(\mathrm{C}-\mathrm{H}), 1637$ $(\mathrm{C}=\mathrm{N}), 1582(\mathrm{Ar} \mathrm{C}=\mathrm{C}), 1172(\mathrm{C}-\mathrm{N}), 729(\mathrm{C}-\mathrm{H}) \mathrm{cm}^{-1}$. HRMS (ESI) calcd for $\mathrm{C}_{48} \mathrm{H}_{51} \mathrm{~B}_{2} \mathrm{~F}_{8} \mathrm{~N}_{10}\left[\mathrm{M}-\mathrm{BF}_{4}^{-}\right]^{+}$ 941.4357, found 941.4376; $\mathrm{C}_{48} \mathrm{H}_{51} \mathrm{BF}_{4} \mathrm{~N}_{10}\left[\mathrm{M}-2 \mathrm{BF}_{4}\right]^{2+}$ 427.2163, found 427.2173; $\mathrm{C}_{48} \mathrm{H}_{51} \mathrm{~N}_{10}\left[\mathrm{M}-3 \mathrm{BF}_{4}^{-}\right]^{3+}$ 255.8099 , found 255.8097 .

三-[2-(3-对硝基苄基- $1 H$-苯并咪唑-2-亚基)乙基]胺 氟硼酸盐(3f): 淡黄色固体, 收率 $77.8 \%$. m.p. 205.7 $206.6{ }^{\circ} \mathrm{C} ;{ }^{1} \mathrm{H}$ NMR $\left(500 \mathrm{MHz}\right.$, DMSO- $\left.d_{6}\right) \delta: 9.84$ (s, $3 \times$ $1 \mathrm{H}), 7.90(\mathrm{~d}, J=8.05 \mathrm{~Hz}, 3 \times 1 \mathrm{H}), 7.87(\mathrm{~d}, J=8.05 \mathrm{~Hz}$, $3 \times 1 \mathrm{H}), 7.65 \sim 7.61(\mathrm{~m}, 3 \times 2 \mathrm{H}), 7.36(\mathrm{~d}, J=7.95 \mathrm{~Hz}, 3 \times$ $2 \mathrm{H}), 7.15(\mathrm{~d}, J=7.9 \mathrm{~Hz}, 3 \times 2 \mathrm{H}), 5.65(\mathrm{~s}, 3 \times 2 \mathrm{H}), 4.52(\mathrm{t}$, $J=6.35 \mathrm{~Hz}, 3 \times 2 \mathrm{H}), 3.30(\mathrm{t}, J=6.35 \mathrm{~Hz}, 3 \times 2 \mathrm{H})$; IR (KBr) v: $3183(\mathrm{C}-\mathrm{H}), 2915(\mathrm{C}-\mathrm{H}), 1602(\mathrm{C}=\mathrm{N}), 1585$ $(\mathrm{Ar}, \mathrm{C}=\mathrm{C}), 1529\left(\mathrm{Ar}-\mathrm{NO}_{2}\right), 1101(\mathrm{C}-\mathrm{N}), 721(\mathrm{C}-\mathrm{H})$ $\mathrm{cm}^{-1}$. HRMS (ESI) calcd for $\mathrm{C}_{48} \mathrm{H}_{45} \mathrm{~B}_{2} \mathrm{~F}_{8} \mathrm{~N}_{10} \mathrm{O}_{6}[\mathrm{M}-$ $\left.\mathrm{BF}_{4}^{-}\right]^{+}$1031.3582, found 1031.3586; $\mathrm{C}_{48} \mathrm{H}_{45} \mathrm{BF}_{4} \mathrm{~N}_{10} \mathrm{O}_{6}$ $\left[\mathrm{M}-2 \mathrm{BF}_{4}\right]^{2+}$ 472.1776, found 472.1767; $\mathrm{C}_{48} \mathrm{H}_{45} \mathrm{~N}_{10} \mathrm{O}_{6}$ $\left[\mathrm{M}-3 \mathrm{BF}_{4}^{-}\right]^{3+} 285.7841$, found 285.7828 .

三-[2-(3-对氯苄基- $1 H$-苯并咪唑-2-亚基)乙基]胺氟 嗍酸盐 $(3 g)$ : 灰白色固体, 收率 80.4\%. m.p. 192.6 $194.1{ }^{\circ} \mathrm{C} ;{ }^{1} \mathrm{H}$ NMR $\left(500 \mathrm{MHz}\right.$, DMSO- $\left.d_{6}\right) \delta: 9.73$ (s, $3 \times$ 1H), $7.94(\mathrm{~d}, J=7.55 \mathrm{~Hz}, 3 \times 1 \mathrm{H}), 7.93(\mathrm{~d}, J=7.5 \mathrm{~Hz}, 3 \times$ $1 \mathrm{H}), 7.67 \sim 7.64(\mathrm{~m}, 3 \times 2 \mathrm{H}), 7.61(\mathrm{~d}, J=8.0 \mathrm{~Hz}, 3 \times 2 \mathrm{H})$, $7.61(\mathrm{~d}, J=7.95 \mathrm{~Hz}, 3 \times 2 \mathrm{H}), 5.74(\mathrm{~s}, 3 \times 2 \mathrm{H}), 4.52(\mathrm{t}, J=$ $6.5 \mathrm{~Hz}, 3 \times 2 \mathrm{H}), 3.30(\mathrm{t}, J=6.5 \mathrm{~Hz}, 3 \times 2 \mathrm{H})$; IR $(\mathrm{KBr}) v$ : $3089(\mathrm{C}-\mathrm{H}), 2906(\mathrm{C}-\mathrm{H}), 1662(\mathrm{C}=\mathrm{N}), 1582(\mathrm{Ar}, \mathrm{C}=$ C), $1172(\mathrm{C}-\mathrm{N}), 728(\mathrm{C}-\mathrm{H}) \mathrm{cm}^{-1}$. HRMS (ESI) calcd for $\mathrm{C}_{48} \mathrm{H}_{45} \mathrm{Cl}_{3} \mathrm{~B}_{2} \mathrm{~F}_{8} \mathrm{~N}_{7}\left[\mathrm{M}-\mathrm{BF}_{4}^{-}\right]^{+}$998.2860, found 998.2841; $\mathrm{C}_{48} \mathrm{H}_{45} \mathrm{Cl}_{3} \mathrm{BF}_{4} \mathrm{~N}_{7}\left[\mathrm{M}-2 \mathrm{BF}_{4}\right]^{2+}$ 455.6415, found 455.6412; $\mathrm{C}_{48} \mathrm{H}_{45} \mathrm{Cl}_{3} \mathrm{~N}_{7}\left[\mathrm{M}-3 \mathrm{BF}_{4}^{-}\right]^{3+}$ 274.7601, found
274.7598.

三-[2-(3-对溴苄基- $1 H$-苯并咪唑-2-亚基)乙基]胺氟 硼酸盐 $(\mathbf{3 h})$ : 白色固体，收率 78.2\%. m.p. 185.6 $186.7^{\circ} \mathrm{C}$; ${ }^{1} \mathrm{H}$ NMR $\left(500 \mathrm{MHz}\right.$, DMSO- $\left.d_{6}\right) \delta$ : 9.75 (s, $3 \times$ $1 \mathrm{H}), 7.94(\mathrm{~d}, J=6.75 \mathrm{~Hz}, 3 \times 1 \mathrm{H}), 7.91(\mathrm{~d}, J=6.7 \mathrm{~Hz}, 3 \times$ $1 \mathrm{H}), 7.66 \sim 7.62(\mathrm{~m}, 3 \times 2 \mathrm{H}), 7.48(\mathrm{~d}, J=6.5 \mathrm{~Hz}, 3 \times 2 \mathrm{H})$, $7.45(\mathrm{~d}, J=6.35 \mathrm{~Hz}, 3 \times 2 \mathrm{H}), 5.74(\mathrm{~s}, 3 \times 2 \mathrm{H}), 4.52(\mathrm{t}, J=$ $6.65 \mathrm{~Hz}, 3 \times 2 \mathrm{H}), 3.29(\mathrm{t}, J=6.65 \mathrm{~Hz}, 3 \times 2 \mathrm{H}) ; \mathrm{IR}(\mathrm{KBr}) v$ : $3052(\mathrm{C}-\mathrm{H}), 2936(\mathrm{C}-\mathrm{H}), 1642(\mathrm{C}=\mathrm{N}), 1577(\mathrm{ArC}=$ C), $1128(\mathrm{C}-\mathrm{N}), 727(\mathrm{C}-\mathrm{H}) \mathrm{cm}^{-1}$. HRMS (ESI) calcd for $\mathrm{C}_{48} \mathrm{H}_{45} \mathrm{Br}_{3} \mathrm{~B}_{2} \mathrm{~F}_{8} \mathrm{~N}_{7}\left[\mathrm{M}-\mathrm{BF}_{4}^{-}\right]^{+}$1134.1323, found 1134.1316; $\mathrm{C}_{48} \mathrm{H}_{45} \mathrm{Br}_{3} \mathrm{BF}_{4} \mathrm{~N}_{7}\left[\mathrm{M}-2 \mathrm{BF}_{4}\right]^{2+}$ 523.5641, found 523.5629; $\mathrm{C}_{48} \mathrm{H}_{45} \mathrm{Br}_{3} \mathrm{~N}_{7}\left[\mathrm{M}-3 \mathrm{BF}_{4}^{-}\right]^{3+} 320.0413$, found 320.0403 .

三-[2-(3-对氟苄基- $1 H$-苯并咪唑-2-亚基)乙基]胺氟 硼酸盐 (3i): 白色固体, 收率 $78.9 \%$. m.p. 188.3 $190.2^{\circ} \mathrm{C} ;{ }^{1} \mathrm{H}$ NMR $\left(500 \mathrm{MHz}\right.$, DMSO- $\left.d_{6}\right) \delta$ : $9.74(\mathrm{~s}, 3 \times$ 1H), $7.96(\mathrm{~d}, J=8.35 \mathrm{~Hz}, 3 \times 1 \mathrm{H}), 7.94(\mathrm{~d}, J=8.3 \mathrm{~Hz}, 3 \times$ $1 \mathrm{H}), 7.67 \sim 7.64(\mathrm{~m}, 3 \times 2 \mathrm{H}), 7.61(\mathrm{~d}, J=8.4 \mathrm{~Hz}, 3 \times 2 \mathrm{H})$, $7.44(\mathrm{t}, J=8.45 \mathrm{~Hz}, 3 \times 2 \mathrm{H}), 5.72(\mathrm{~s}, 3 \times 2 \mathrm{H}), 4.51(\mathrm{t}, J=$ $6.5 \mathrm{~Hz}, 3 \times 2 \mathrm{H}), 3.31(\mathrm{t}, J=6.5 \mathrm{~Hz}, 3 \times 2 \mathrm{H}) ; \mathrm{IR}(\mathrm{KBr}) v$ : $3069(\mathrm{C}-\mathrm{H}), 2916(\mathrm{C}-\mathrm{H}), 1631(\mathrm{C}=\mathrm{N}), 1572(\mathrm{Ar}, \mathrm{C}=$ C), $1163(\mathrm{C}-\mathrm{N}), 731(\mathrm{C}-\mathrm{H}) \mathrm{cm}^{-1}$; HRMS (ESI) calcd for $\mathrm{C}_{48} \mathrm{H}_{45} \mathrm{~B}_{2} \mathrm{~F}_{11} \mathrm{~N}_{7}\left[\mathrm{M}-\mathrm{BF}_{4}^{-}\right]^{+}$950.3747, found 950.3760; $\mathrm{C}_{48} \mathrm{H}_{45} \mathrm{BF}_{7} \mathrm{~N}_{7}\left[\mathrm{M}-2 \mathrm{BF}_{4}^{-}\right]^{2+} 431.6859$, found 431.6852; $\mathrm{C}_{48} \mathrm{H}_{45} \mathrm{~F}_{3} \mathrm{~N}_{7}\left[\mathrm{M}-3 \mathrm{BF}_{4}^{-}\right]^{3+}$ 258.7896, found 258.7888 .

三-\{2-[3-(吲哚-3-甲基-1-(苯基磺酰基)- $1 H$-苯并咪 唑-2-亚基]乙基 $\}$ 胺氟硼酸盐 $(\mathbf{3 j})$ : 黄色固体, 收率 71.3\%. m.p. $208.1 \sim 209.3{ }^{\circ} \mathrm{C}$; ${ }^{1} \mathrm{H}$ NMR $(500 \mathrm{MHz}$, DMSO- $\left.d_{6}\right) \delta: 9.76(\mathrm{~s}, 3 \times 1 \mathrm{H}), 8.32(\mathrm{~d}, J=4.85 \mathrm{~Hz}, 3 \times$ $1 \mathrm{H}), 7.93(\mathrm{~d}, J=8.15,3 \times 1 \mathrm{H}), 7.78 \sim 7.70(\mathrm{~m}, 3 \times 2 \mathrm{H})$, $7.72 \sim 7.70(\mathrm{~m}, 3 \times 5 \mathrm{H}), 6.97 \sim 6.90(\mathrm{~m}, 3 \times 5 \mathrm{H}), 5.61(\mathrm{~s}$, $3 \times 2 \mathrm{H}), 4.49(\mathrm{t}, J=6.75 \mathrm{~Hz}, 3 \times 2 \mathrm{H}), 3.27(\mathrm{t}, J=6.75 \mathrm{~Hz}$, $3 \times 2 \mathrm{H})$; IR $(\mathrm{KBr}) v: 3092(\mathrm{C}-\mathrm{H}), 2945(\mathrm{C}-\mathrm{H}), 1634$ $(\mathrm{C}=\mathrm{N}), 1562(\mathrm{Ar}, \mathrm{C}=\mathrm{C}), 1128(\mathrm{C}-\mathrm{N}), 1186(\mathrm{O}=\mathrm{S}=\mathrm{O})$, $725(\mathrm{C}-\mathrm{H}) \mathrm{cm}^{-1}$. HRMS (ESI) calcd for $\mathrm{C}_{72} \mathrm{H}_{63} \mathrm{~B}_{2^{-}}$ $\mathrm{F}_{8} \mathrm{~N}_{10} \mathrm{O}_{6} \mathrm{~S}_{3}\left[\mathrm{M}-\mathrm{BF}_{4}^{-}\right]^{+}$1433.4153, found 1433.4148; $\mathrm{C}_{72} \mathrm{H}_{63} \mathrm{BF}_{4} \mathrm{~N}_{10} \mathrm{O}_{6} \mathrm{~S}_{3} \quad\left[\mathrm{M}-2 \mathrm{BF}_{4}^{-}\right]^{2+}$ 673.2061, found 673.2073; $\mathrm{C}_{72} \mathrm{H}_{63} \mathrm{~N}_{10} \mathrm{O}_{6} \mathrm{~S}_{3}\left[\mathrm{M}-3 \mathrm{BF}_{4}^{-}\right]^{3+}$ 419.8031, found 419.8027.

三-\{2-[3-(噻吩-2-甲基)- $1 H$-苯并咪唑-2-亚基]乙基\} 胺氟嗍酸盐(3k): 灰色固体, 收率 73.5\%. m.p. 191.5 $192.9{ }^{\circ} \mathrm{C} ;{ }^{1} \mathrm{H}$ NMR (500 MHz, DMSO- $\left.d_{6}\right) \delta$ : 9.74 (s, $3 \times$ $1 \mathrm{H}), 8.11(\mathrm{~d}, J=8.2 \mathrm{~Hz}, 3 \times 1 \mathrm{H}), 7.94(\mathrm{~d}, J=8.25 \mathrm{~Hz}, 3 \times$ 
1H), $7.72 \sim 7.64(\mathrm{~m}, 3 \times 2 \mathrm{H}), 7.56 \sim 7.54(\mathrm{~m}, 3 \times 1 \mathrm{H}), 7.39$ $(\mathrm{d}, J=3.05 \mathrm{~Hz}, 3 \times 1 \mathrm{H}), 7.04 \sim 7.03(\mathrm{~m}, 3 \times 1 \mathrm{H}), 5.97(\mathrm{~s}$, $3 \times 2 \mathrm{H}), 4.50(\mathrm{t}, J=6.35 \mathrm{~Hz}, 3 \times 2 \mathrm{H}), 3.24(\mathrm{t}, J=6.3 \mathrm{~Hz}$, $3 \times 2 \mathrm{H})$; IR (KBr) v: $3188(\mathrm{C}-\mathrm{H}), 2901(\mathrm{C}-\mathrm{H}), 1684$ $(\mathrm{C}=\mathrm{N}), 1527(\mathrm{Ar}, \mathrm{C}=\mathrm{C}), 1118(\mathrm{C}-\mathrm{N}), 747(\mathrm{C}-\mathrm{H}), 648$ $(\mathrm{C}-\mathrm{S}) \mathrm{cm}^{-1}$. HRMS (ESI) calcd for $\mathrm{C}_{42} \mathrm{H}_{42} \mathrm{~B}_{2} \mathrm{~F}_{8} \mathrm{~N}_{7} \mathrm{~S}_{3}$ $\left[\mathrm{M}-\mathrm{BF}_{4}^{-}\right]^{+}$914.2722, found 914.2717; $\mathrm{C}_{42} \mathrm{H}_{42} \mathrm{BF}_{4} \mathrm{~N}_{7} \mathrm{~S}_{3}$ $\left[\mathrm{M}-2 \mathrm{BF}_{4}^{-}\right]^{2+} 413.6346$, found 413.6349; $\mathrm{C}_{42} \mathrm{H}_{42} \mathrm{~N}_{7} \mathrm{~S}_{3}$ $\left[\mathrm{M}-3 \mathrm{BF}_{4}^{-}\right]^{3+} 246.7555$, found 246.7543 .

三-\{2-[3-(吡啶-2-甲基)- $1 H$-苯并咪唑]乙基 $\}$ 胺氟硼 酸盐 $(31)$ : 灰白色固体, 收率 $72.8 \%$. m.p. 185.2 $186.7^{\circ} \mathrm{C} ;{ }^{1} \mathrm{H}$ NMR $\left(500 \mathrm{MHz}, \mathrm{DMSO}-d_{6}\right) \delta: 9.80$ (s, $3 \times$ $1 \mathrm{H}), 9.05$ (d, $J=7.2 \mathrm{~Hz}, 3 \times 1 \mathrm{H}), 7.98(\mathrm{~d}, J=8.2 \mathrm{~Hz}, 3 \times$ $1 \mathrm{H}), 7.88(\mathrm{~d}, J=8.15 \mathrm{~Hz}, 3 \times 1 \mathrm{H}), 7.67(\mathrm{t}, J=7.45 \mathrm{~Hz}, 3 \times$ $1 \mathrm{H}), 7.61$ (t, $J=7.8 \mathrm{~Hz}, 3 \times 1 \mathrm{H}), 7.50(\mathrm{~d}, J=7.1 \mathrm{~Hz}, 3 \times$ $1 \mathrm{H}), 7.34$ (t, $J=7.6 \mathrm{~Hz}, 3 \times 1 \mathrm{H}), 7.04$ (d, $J=8.2 \mathrm{~Hz}, 3 \times$ $1 \mathrm{H}), 5.64(\mathrm{~s}, 3 \times 2 \mathrm{H}), 4.50(\mathrm{t}, J=6.65 \mathrm{~Hz}, 3 \times 2 \mathrm{H}), 3.23(\mathrm{t}$, $J=6.65 \mathrm{~Hz}, 3 \times 2 \mathrm{H})$; IR (KBr) v: $3125(\mathrm{C}-\mathrm{H}), 2915(\mathrm{C}-$ $\mathrm{H}), 1623(\mathrm{C}=\mathrm{N}), 1537(\mathrm{Ar}, \mathrm{C}=\mathrm{C}), 1138(\mathrm{C}-\mathrm{N}), 739$ $(\mathrm{C}-\mathrm{H}) \mathrm{cm}^{-1}$. HRMS (ESI) calcd for $\mathrm{C}_{45} \mathrm{H}_{45} \mathrm{~B}_{2} \mathrm{~F}_{8} \mathrm{~N}_{10}[\mathrm{M}-$ $\left.\mathrm{BF}_{4}{ }^{-}\right]$899.3887, found 899.3886; $\mathrm{C}_{45} \mathrm{H}_{45} \mathrm{BF}_{4} \mathrm{~N}_{10} \quad[\mathrm{M}-$ $\left.2 \mathrm{BF}_{4}^{-}\right]^{2+} 406.1929$, found 406.1921; $\mathrm{C}_{45} \mathrm{H}_{45} \mathrm{~N}_{10}[\mathrm{M}-$ $\left.3 \mathrm{BF}_{4}^{-}\right]^{3+} 241.7943$, found 241.7928 .

三-[2-(3-戊基- $1 H$-苯并咪唑-2-亚基)乙基]胺氟硼酸 盐(3m): 灰色固体, 收率 $72.8 \%$. m.p. $178.4 \sim 179.6{ }^{\circ} \mathrm{C}$; ${ }^{1} \mathrm{H}$ NMR $\left(500 \mathrm{MHz}, \mathrm{DMSO}-d_{6}\right) \delta: 9.56(\mathrm{~s}, 3 \times 1 \mathrm{H}), 8.07$ (d, $J=7.25 \mathrm{~Hz}, 3 \times 1 \mathrm{H}), 8.02(\mathrm{~d}, J=7.25 \mathrm{~Hz}, 3 \times 1 \mathrm{H})$, $7.65 \sim 7.63(\mathrm{~m}, 3 \times 2 \mathrm{H}), 4.59(\mathrm{t}, J=6.65 \mathrm{~Hz}, 3 \times 2 \mathrm{H}), 4.51$ $(\mathrm{t}, J=7.5 \mathrm{~Hz}, 3 \times 2 \mathrm{H}), 3.25(\mathrm{t}, J=6.6 \mathrm{~Hz}, 3 \times 2 \mathrm{H}), 1.85$ (q, $3 \times 2 \mathrm{H}), 1.31 \sim 1.28(\mathrm{~m}, 3 \times 2 \mathrm{H}), 1.28 \sim 1.25(\mathrm{~m}, 3 \times 2 \mathrm{H})$, $0.85(\mathrm{t}, J=2.55 \mathrm{~Hz}, 3 \times 3 \mathrm{H})$; IR $(\mathrm{KBr}) v: 3110(\mathrm{C}-\mathrm{H})$, $2928(\mathrm{C}-\mathrm{H}), 1671(\mathrm{C}=\mathrm{N}), 1552(\mathrm{Ar}, \mathrm{C}=\mathrm{C}), 1148(\mathrm{C}-$ $\mathrm{N}), 726(\mathrm{C}-\mathrm{H}) \mathrm{cm}^{-1}$. HRMS (ESI) calcd for $\mathrm{C}_{45} \mathrm{H}_{66} \mathrm{~B}_{2} \mathrm{~F}_{8} \mathrm{~N}_{7}\left[\mathrm{M}-\mathrm{BF}_{4}^{-}\right]^{+}$878.5438, found 878.5431; $\mathrm{C}_{45} \mathrm{H}_{66} \mathrm{BF}_{4} \mathrm{~N}_{7}\left[\mathrm{M}-2 \mathrm{BF}_{4}^{-}\right]^{2+}$ 395.7704, found 395.7693; $\mathrm{C}_{45} \mathrm{H}_{66} \mathrm{~N}_{7}\left[\mathrm{M}-3 \mathrm{BF}_{4}^{-}\right]^{3+}$ 243.8460, found 243.8461 .

三-[2-(3-庚基- $1 H$-苯并咪唑-2-亚基)乙基]胺氟硼酸 盐(3n): 灰色固体, 收率 71.2\%. m.p. 183.2 184.9 ${ }^{\circ} \mathrm{C}$; ${ }^{1} \mathrm{H}$ NMR (500 Hz, DMSO-d $) \delta: 9.64(\mathrm{~s}, 3 \times 1 \mathrm{H}), 8.13$ (d, $J=7.85 \mathrm{~Hz}, 3 \times 1 \mathrm{H}), 8.05(\mathrm{~d}, J=7.9 \mathrm{~Hz}, 3 \times 1 \mathrm{H}), 7.83 \sim$ $7.81(\mathrm{~m}, 3 \times 2 \mathrm{H}), 4.62(\mathrm{t}, J=6.55 \mathrm{~Hz}, 3 \times 2 \mathrm{H}), 4.54(\mathrm{t}, J=$ $7.45 \mathrm{~Hz}, 3 \times 2 \mathrm{H}), 3.36(\mathrm{t}, J=6.5 \mathrm{~Hz}, 3 \times 2 \mathrm{H}), 1.98(\mathrm{q}, 3 \times$ $2 \mathrm{H}), 1.38 \sim 1.35(\mathrm{~m}, 3 \times 2 \mathrm{H}), 1.36 \sim 1.32(\mathrm{~m}, 3 \times 6 \mathrm{H}), 0.88$ (t, $J=2.25 \mathrm{~Hz}, 3 \times 3 \mathrm{H})$; IR (KBr) v: $3029(\mathrm{C}-\mathrm{H}), 2923$ $(\mathrm{C}-\mathrm{H}), 1643(\mathrm{C}=\mathrm{N}), 1556(\mathrm{Ar}, \mathrm{C}=\mathrm{C}), 1218(\mathrm{C}-\mathrm{N})$,
$729(\mathrm{C}-\mathrm{H}) \mathrm{cm}^{-1}$. HRMS (ESI) calcd for $\mathrm{C}_{51} \mathrm{H}_{78} \mathrm{~B}_{2} \mathrm{~F}_{8} \mathrm{~N}_{7}$ $\left[\mathrm{M}-\mathrm{BF}_{4}^{-}\right]^{+}$962.6377, found 962.6380; $\mathrm{C}_{51} \mathrm{H}_{78} \mathrm{BF}_{4} \mathrm{~N}_{7}$ $\left[\mathrm{M}-2 \mathrm{BF}_{4}^{-}\right]^{2+} 437.8174$, found 437.8156; $\mathrm{C}_{51} \mathrm{H}_{78} \mathrm{~N}_{7}[\mathrm{M}-$ $\left.3 \mathrm{BF}_{4}^{-}\right]^{3+} 262.8773$, found 262.8754 .

致谢 活性耖选试验由国家新药耖选中心协助测定, 在 此对他们的帮助表示诚挚的谢意.

辅助材料(Supporting Information) 提供了所有目标 化合物的核磁共振氢谱和高分辨质谱. 这些材料可以免 费从本刊网站(http://sioc-journal.cn/)上下载.

\section{References}

[1] Song, L.; Xie, Y. Y.; Wang, H. Z. Chin. J. Med. Chem. 2000, 92. (in Chinese).

(宋琳, 谢毓元, 王惠贞，中国药物化学杂志, 2000, 92.)

[2] Meng, J. P.; Lu, Y. H.; Halqam, I.; Zhou, C. H. Chin. J. Biochem. Pharmacol. 2008, 29, 418. (in Chinese).

(孟江平, 卢一卉, 海力且木 - 依不那音, 周成合, 中国生化药物 杂志, 2008, 29, 418.)

[3] Guo. Y. C.; Zhou, L. H.; Qiao. Z. P. Chin. J. Inorg. Chem. 2006 134 (in Chinese)

(郭应臣, 卓立宏, 乔占平, 无机化学学报, 2006, 134.)

[4] Ören, I.; Temiz, Ö.; Yalıın, I.; Şener, E.; Altanlar, N. Eur. Pharm. Sci. 1989, 7, 153.

[5] Klimešová, V.; Kočía, J.; Pour, M.; Stachel, J.; Waisser, K.; Kaustová, J. Eur. Med. Chem. 2002, 33, 137.

[6] Ansari, K. F.; Lal, C. Eur. J. Med. Chem. 2009, 44, 2294.

[7] Rajakumara, P.; Sekara, K.; Shanmugaiahb, V.; Mathivananb, N. Bioorg. Med. Chem. Lett. 2008, 18, 4416.

[8] Beaulieu, P. L.; Bousquet, Y.; Gauthier, J.; Gillard, J.; Marquis, M.; McKercher, G.; Pellerin, C.; Valois, S.; Kukolj, G. J. Med. Chem. 2004, 47, 6884 .

[9] Hirashima, S.; Suzuki, T.; Ishida, T.; Noji, S.; Yata, S.; Ando, I.; Komatsu, M.; Ikeda, S.; Hashimoto, H. T. J. Med. Chem. 2006, 49, 4721.

[10] Asthana, S.; Shukla, S.; Vargiu, A. V. Biochemistry 2013, 52, 3752.

[11] Li, Y. F.; Wang, G. F.; He, P. L.; Huang, W. G.; Zhu, F. H. ; Gao, H. Y.; Tang, W.; Luo, Y.; Feng, C. L.; Shi, Li. P.; Ren, Y. D.; Lu, W.; Zuo, J. P. J. Med. Chem. 2006, 49, 4790.

[12] Fang, X. P.; Mei, X. G. Foreign Med. Sci. 2005, 32, 115 (in Chinese). (方学平, 梅兴国, 国外医学, 2005, 32, 15.)

[13] Combs, A. P.; Zhu, W. Y.; Crawley, M. L.; Glass, B.; Polam, P.; Sparks, R. B.; Modi, D.; Takvorian, A.; McLaughlin, E.; Yue, E. W.; Wasserman, Z.; Bower, M.; Wei, M.; Rupar, M.; Ala, P. J.; Reid, B. M.; Ellis, D.; Gonneville, L.; Emm, T.; Taylor, N.; Yeleswaram, S.; Li Y. L.; Richard, W.; Timothy, C. B.; Gregory, H.; Phillip, C. C.; Liu, B. M. J. Med. Chem. 2006, 49, 3774.

[14] Ong, J. X.; Yap, C. W.; Ang, W. H. Inorg. Chem. 2012, 51, 12483.

[15] Erion, M. D.; Dang, Q.; Reddy, M. R.; Kasibhatla, S. R.; Huang, J. W.; Lipscomb, W. N.; Poelje, P. D. J. Am. Chem. Soc. 2008, 129, 15480 .

[16] Dang, Q.; Kasibhatla, S. R.; Xiao, W.; Liu, Y.; DaRe, J.; Taplin, F.; Reddy, K. R.; Scarlato, G. R.; Gibson, T.; Poelje, P. D.; Potter, S. C.; Erion, M. D. J. Med. Chem. 2010, 53, 441.

[17] Settimo, F. D.; Primofiore, G.; Settimo, A. D. J. Med. Chem. 2001, 
44, 4359 .

[18] Ramla, M. M.; Omar, M. A.; El-Khamry, A-M. M.; El-Diwani, H. I. Bioorg. Med. Chem. 2006, 14, 7324.

[19] Rewcastle, G. W.; Gamage, S. A.; Flanagan, J. U. J. Med. Chem. 2011, 54, 105.

[20] Lavecchia, A.; Giovanni, C. D.; Pesapane, A.; Montuori, N.; Ragno, P.; Martucci, N. M.; Masullo, M.; Vendittis, E. D.; Novellino, E. J. Med. Chem. 2012, 55, 4142.

[21] Zhang, C. L.; Wang, X.; Guo, Y.; Wu, Y. F.; Gao, L. N.; Sun, L. J. Chai, J. H.; Zhu, C. A. Chin. J. Org. Chem. 2014, 34, 2331 (in Chinese).

(张成路, 王雪, 国阳, 吴一非, 高丽娜, 孙丽杰, 柴金华, 朱长 安, 有机化学, 2014, 34, 2331.)

[22] Vladislav, Y. K.; Igor, B. K. ; Vyacheslav, Y. S. Heteroat. Chem. 2005, 16, 492.

[23] Zhang, C. L.; Sun, L. J.; Wu, F. Y.; Qu, R. F.; Zhu, C. A.; Wang, X.; Chai, J. H.; Guo, Y.; Hu, X. Chem. J. Chin. Univ. 2014, 35, 1445 (in Chinese).

(张成路, 孙立杰, 武飞宇, 曲瑞峰, 朱长安, 王雪, 柴金华, 国 阳，胡雪，高等学校化学学报, 2014, 35, 1445.)

[24] Fukuyama, Y.; Tanimura, R.; Maeda, K. Anal. Chem. 2012, 84, 4237.

[25] Singh, N.; Jang, D. O. Org. Lett. 2007, 9, 1991.

[26] Martinez, M.; Scancenon, F. Chem. Rev 2003, 103, 4419.

[27] Kim, Y. H.; Sun, G. Text. Res. J. 2001, 71, 318.

[28] Kar, C.; Basu, A.; Das, G. Tetrahedron 2012, 53, 4754.

[29] Zhang, Y. N.; Zhang, W.; Hong, D.; Shi, L.; Shen, Q.; Li, J. Y.; Li, J.; Hua, L. H. Bioorg. Med. Chem. 2008, 16, 8697.
[30] Huang, W. G.; Jiang, Y. Y.; Li, Q.; Li, J.; Li, J. Y.; Lu, W.; Cai, J. C. Tetrahedron 2005, 61, 1863.

[31] Sun, L. P.; Shen, Q.; Piao, H. H.; Ma, W. P.; Gao, L. X.; Zhang, W.; Nan, F. J.; Li, J.; Piao, H. R. Eur. J. Med. Chem. 2011, 46, 3630.

[32] Alam, M. M.; Nazreen, S.; Haider, S.; Shafi, S.; Yar, M. S.; Hamid, H.; Alam, M. S. Pharm. Chem. Life Sci. 2011, 45, 11

[33] Khatri, V. K.; Chahar, M.; Pavani, K.; Pandey, P. S. J. Org. Chem. 2007, 72, 10224.

[34] Boye, B.; Keramanea, E. M.; Monterob, J. L.; Roquea, J. P. Synth. Commun. 1998, 28, 1737.

[35] Wang, Z. T.; Zhu, L.; Yin, F.; Su, Z. Q.; Li, Z. D.; Li, C. Z. J. Am. Chem. Soc. 2012, 134, 4258.

[36] Matsuo, J.; Iida, D.; Yamanakaa, H.; Mukaiyamaa, T. Tetrahedron 2003, 59, 6739.

[37] Sun, J.; Feng, X. J.; Zhao, Z. R.; Yamamoto, Y.; Bao, M. Tetrahedron 2014, 70, 7166.

[38] Niknam, K.; Kiasat, A. R.; Kazemi, F.; Hossienia, A. Phosphorus, Sulfur, Silicon Relat. Elem.2003, 178, 1385.

[39] Li, Z.; Sheng, C.; Qiu, H.; Zhang, Y. The New J. Org. Synth. 2009, $39,412$.

[40] Zhang, J.; Cui, H. L.; Hojo, M.; Shuang, S. M.; Dong, C. Bioorg. Med. Chem. Lett. 2012, 22, 343.

[41] Caliendoa, G.; Fiorinoa, F.; Perissuttia, E.; Severinoa, B.; Gessib, S.; Cattabrigab, E.; Boreab, P. A.; Santagadaa, V. Eur. J. Med. Chem. 2001, 36, 873.

[42] Boger, D. L.; Palanki, M. S. S. J. Am. Chem. Soc. 1992, 114, 9318.

[43] Tan, H. H.; Ong, W. M.; Lai, S. H. Singapore Med. J. 2007, 48, 582.

(Qin, X.) 